\title{
Reação inflamatória e resposta imune (R.I) humoral induzida pelas vacinas comerciais reprodutivas contendo diferentes adjuvantes
}

Camila Costa Baccili[a]", Camila Cecília Martin ${ }^{[a]}$,Jean Silva Ramos ${ }^{[a]}$, Karen Nascimento da Silva[ ${ }^{[a]}$, Luciandra Macedo de Toledo ${ }^{[b]}$, Edviges Maristela Pituco ${ }^{[c]}$ Viviani Gomes ${ }^{[a]}$

\footnotetext{
[a] Faculdade de Medicina Veterinária e Zootecnia, Universidade de São Paulo(USP), São Paulo, SP, Brasil

${ }^{[b]}$ APTA - Secretaria da Agricultura e Abastecimento, Instituto de Zootecnia, Nova Odessa, SP, Brasil

[c] Laboratório de Viroses de Bovídeos, Instituto Biológico, São Paulo, SP, Brasil
}

*Autor correspondente

e-mail: camila.rcosta@usp.br

\section{Resumo}

As vacinas reprodutivas brasileiras contendo o BVDV são inativadas e induzem R.I de baixa intensidade e curta duração. Os adjuvantes podem potencializar a R.I induzida pela vacinação, pela atração e captação dos antígenos pelas células apresentadoras de antígenos (APC's) durante a resposta inflamatória no ponto de aplicação. Neste contexto, o objetivo desta pesquisa foi avaliar a reação inflamatória e R.I humoral induzida por três vacinas reprodutivas contra o BVDV e BoHV-1 contendo diferentes tipos de adjuvantes. Foram selecionadas 35 novilhas Holandesas, entre 15 - 24 meses, distribuídas em quatro grupos de acordo com os adjuvantes das vacinas: hidróxido de alumínio (Halum, $n=9$ ), oleoso (Oleoso, $n=10$ ), mistura de saponina, colesterol e amphigen (Prezent $A, n=10$ ), controle (salina, $n=6$ ). As novilhas receberam duas doses das vacinas, com intervalo de 21 dias, por via subcutânea. A inflamação local foi avaliada pela dor, mensuração da espessura cutânea e imagens termográficas, além de dosagem da haptoglobina (HP) sérica. A inflamação foi avaliada 0, 6, 24, 48, 72 e 168 horas (h) após a aplicação da $1^{\text {a }}$ e $2^{\text {a }}$ dose das vacinas. A produção de anticorpos (ACs) neutralizantes foi determinada no D0, D21 e D42. A estatística foi feita pelo qui-quadrado e oneway ANOVA. A dor foi mais frequente nos animais vacinados com o adjuvante oleoso e Prezent A. Todos os animais vacinados apresentaram aumento de volume local $24 \mathrm{~h}$ após a $1^{\mathrm{a}}$ dose, observando-se maior reação nas novilhas vacinadas com adjuvante oleoso $\left(61 \mathrm{~mm}^{2}\right)$ em relação ao Halum $\left(27 \mathrm{~mm}^{2}\right)$, Prezent A $\left(30 \mathrm{~mm}^{2}\right)$ e controle $\left(10 \mathrm{~mm}^{2}\right)$. Após a $2^{\underline{a}}$ dose, a reatividade foi maior para o Prezent A $\left(60 \mathrm{~mm}^{2}\right)$ em relação ao grupo Oleoso $\left(44 \mathrm{~mm}^{2}\right)$, Halum $\left(34 \mathrm{~mm}^{2}\right)$ e Controle $\left(9 \mathrm{~mm}^{2}\right)$. A temperatura média no ponto de aplicação foi semelhante entre os grupos vacinados, porém diferentes do controle. Os valores de HP foram maiores nas novilhas Prezent $\mathrm{A}$ (4 a $7 \mathrm{mg} / \mathrm{dL})$ em relação às demais vacinas $(1 \mathrm{mg} / \mathrm{dL})$ às 24 h após $1^{\underline{a}}$ e $2^{\underline{a}}$ dose. Em 
relação à produção final de Acs ( $\log _{2}$ ) contra BVDV (D42), pôde-se verificar perfis semelhantes entre Prezent $A$ $(4,8)$ e Halum $(4,7)$. Diferenças entre os títulos de ACs para BVDV foram observadas para a vacina oleosa (1) e controle (0) em relação àquela que continha o adjuvante a Prezent A. Em relação ao BoHV-1 (D42), observouse diferença entre todas a vacinas, observando-se melhor resposta nas novilhas vacinadas com o Prezent $\mathrm{A}$ $\left(\log _{2}=5,6\right)$, comparado ao Halum ( $\left.\log _{2}=3,7\right)$, Oleoso $\left(\log _{2}=3,0\right)$ e controle $(0)$. A vacina oleosa induziu reação local exacerbada com baixa/nula produção de ACs neutralizantes para BVDV. Em geral, a formulação das vacinas contendo Halum e Prezent A promoveram maior resposta imune humoral induzida pela vacinação. 